\section{Stripping paintings of their secrets with hyperspectral imaging}

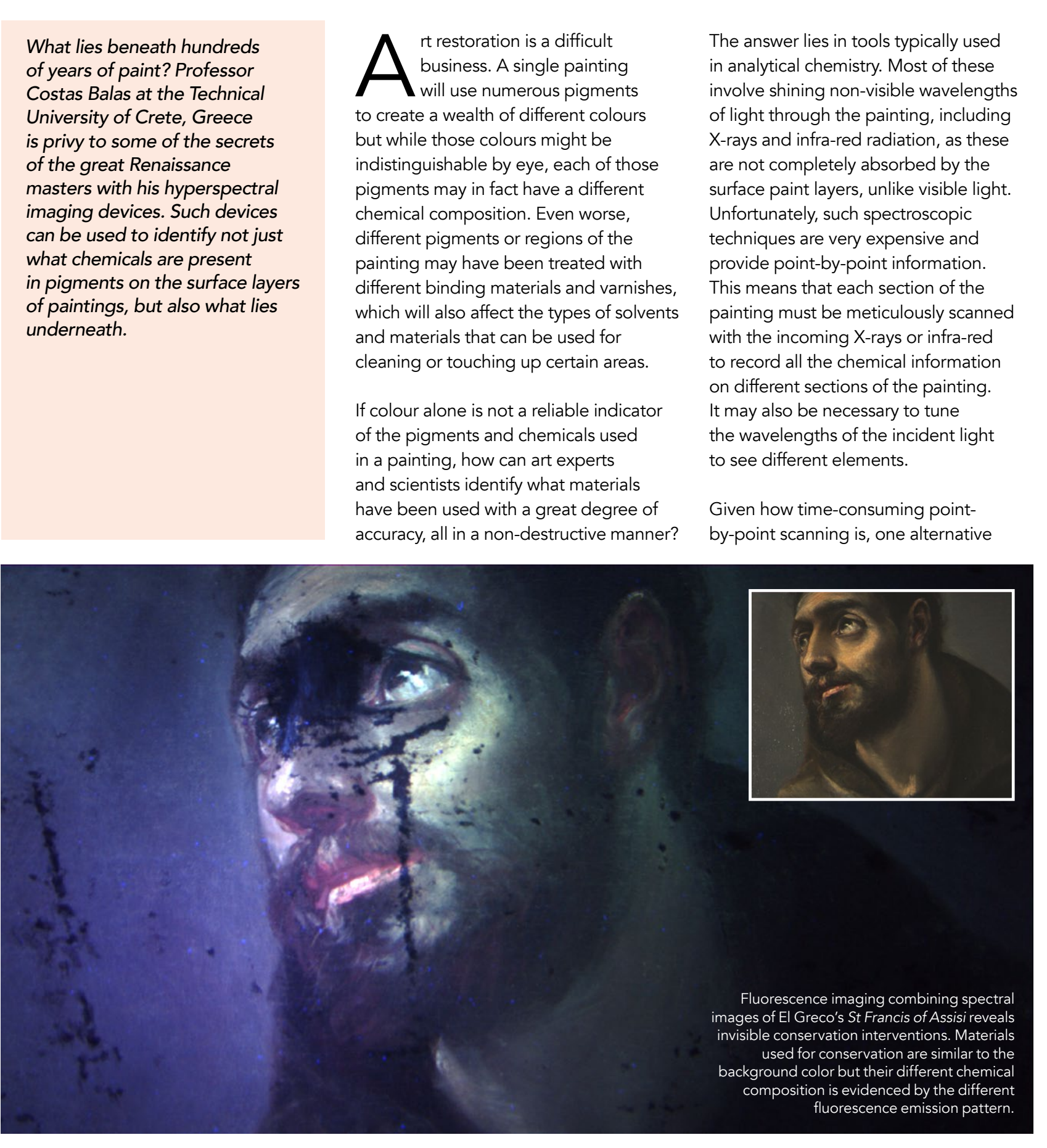

approach is to combine spectroscopic methods with imaging methods. accurately determining which elements are present in a particular region but imaging methods have very high spatial resolution and can therefore disentangle differences in even small, detailed areas of the painting. This combination is known as hyperspectral imaging and Professo Costas Balas at the Technical University of Crete, Greece and his research group are experts in improving and developing hyperspectral imaging technologies for both medical

HYPERSPECTRAL DEVICES Professor Balas's interest in Professor Balas's interest in
hyperspectral imaging was originally motivated by his work in the field of cancer diagnosis and biomedicine. Here, there is a need for devices that can successfully and accurately profile and image tumours within the body as part of the diagnostic process but there are a number of features of the technique that have meant it can be applied to a diverse range of applications, including art restoration and identification.

A hyperspectral imaging device works by simultaneously recording spectral and spatial inages to make three-d mensional data sets. A spatial image is lke a photograph that shows in the fram Howerer as the spectral information can only be obtained for a limited number of wavelengths per spatial image, this means only certain coloured objects are recorded in a single image, so it would be like taking a photograph which would only show green objects. To make up a full threedimensional image, another photograph would be taken, that would only show blue objects, then red objects and so forth until all the wavelengths of interest had been covered and the full scene could be created.

In practice, as the human body and paint absorb visible light, the imaging ranges of the hyperspectralinaging
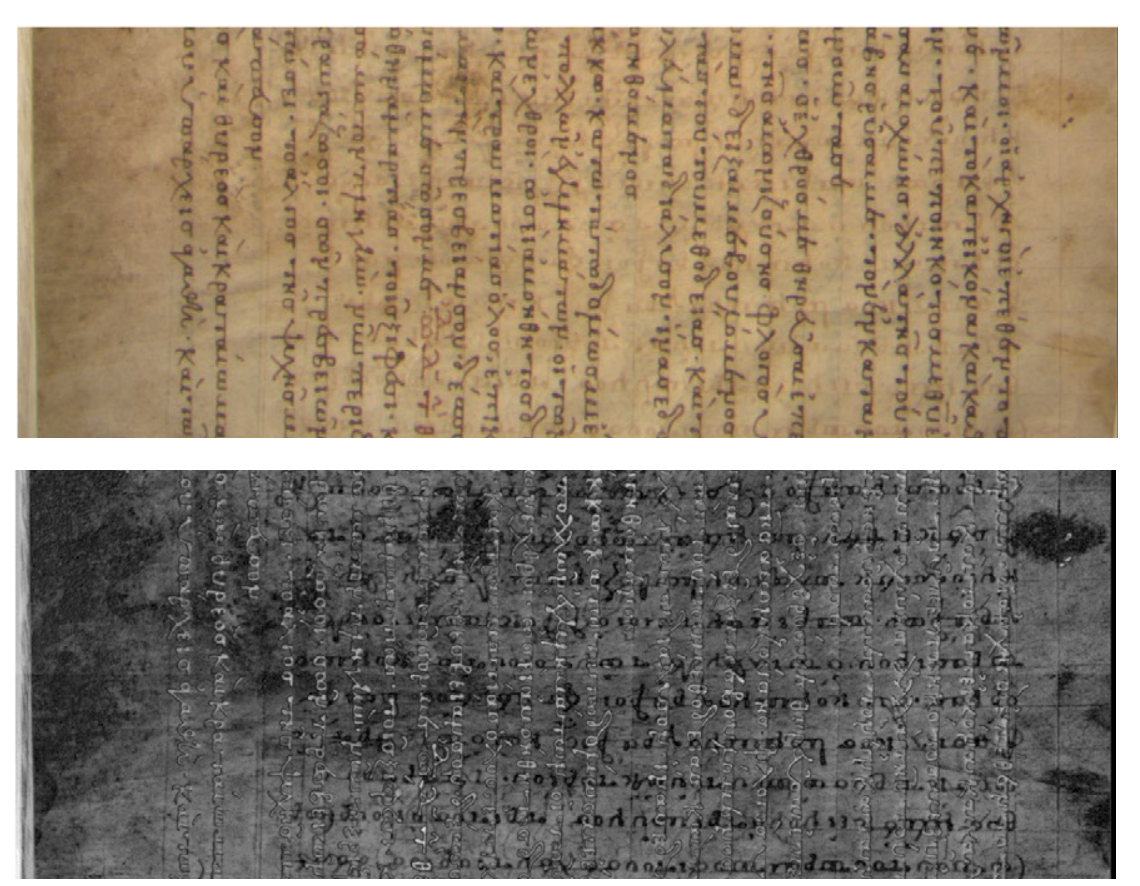

Hyperspectral analysis of the palimpsest (72 verso) (Mount Athos Monasteries-Greece). Regular colour

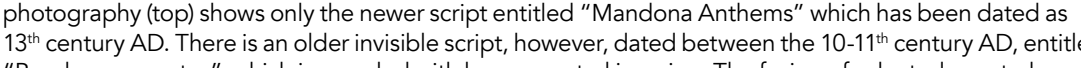
images made the visualisation of both the new (vertical script) and the old (horizontal script) possible.

The team found a number of surprising results that only hyperspectral imaging could have unearthed.

the infra-red. Using these wavelengths of light in the infra-red allows Professor Balas to peel back the layers of paint The flexibility of the wavelengths that The flexibility of the wavelengths that
can be used with hyperspectral imaging has many advantages for dealing with complex materials such as paintings. for identifying colours and pigments,

with ultraviolet and infra-red detection that provide more definitive chemical assignments and are sensilive not just

\section{SEEING THE CANVAS}

Professor Balas and his team have been able to use this technology to study mónikos Theotokópoulos's,

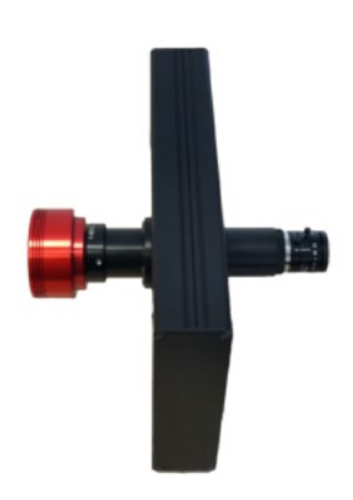

otherwise known as El Greco,

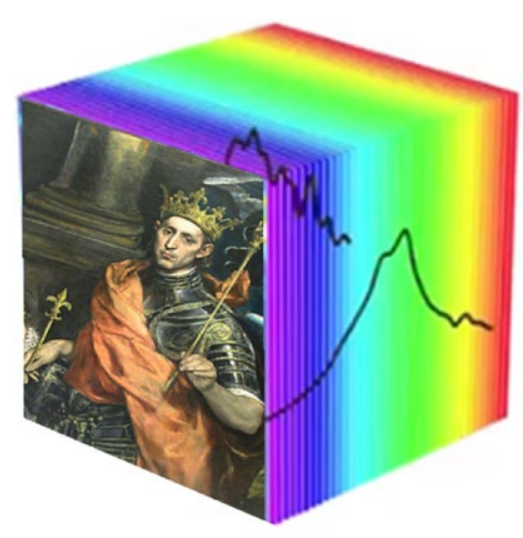

The hyperspectral camera, developed by Professoro Balas and his team, (leff) captures a series of

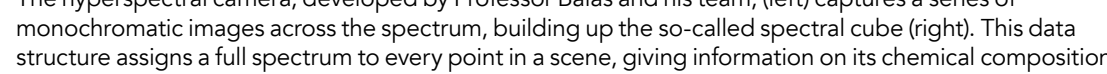



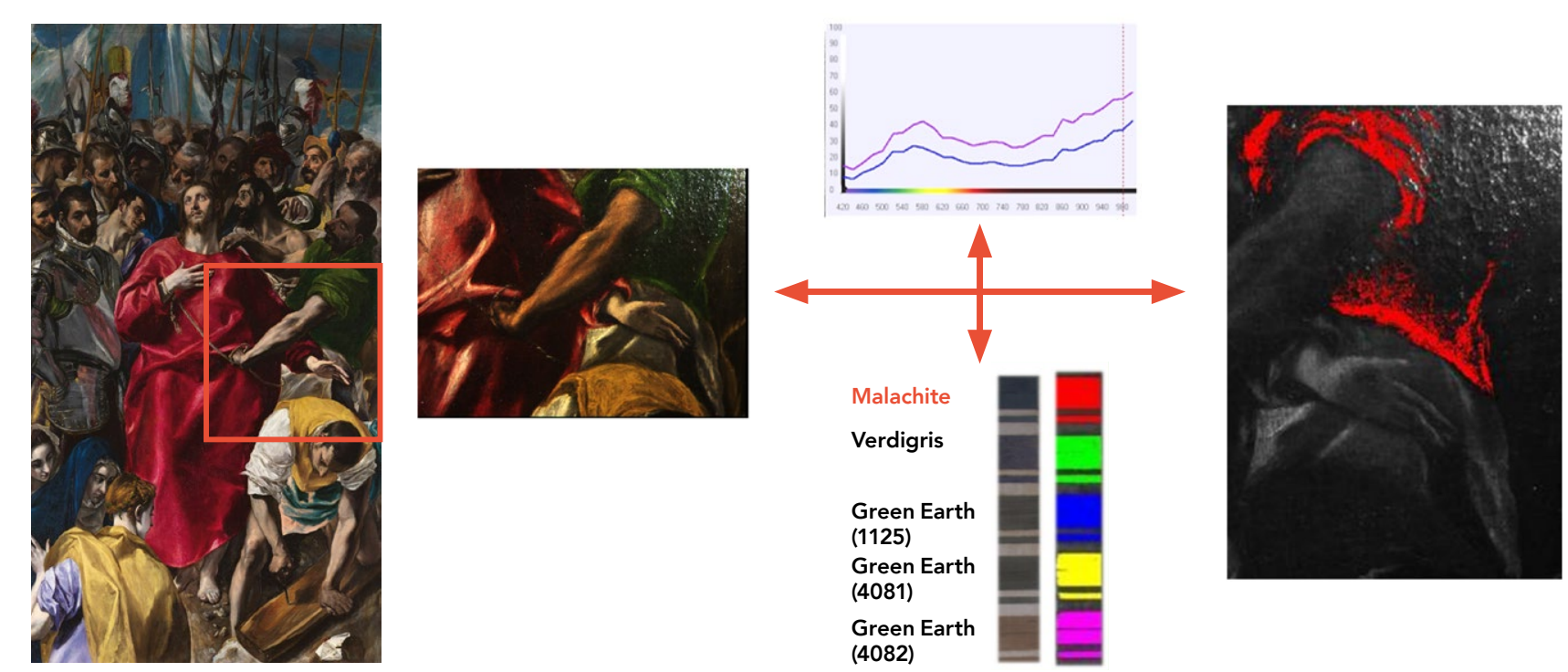

Hyperspectral analysis of "EI Espolio" by Jorge Manuel (EI Greco's son). Spectral mapping of a detail, shown in the red frame, combined with machine learning
methods allows for the identification of malachite pigment non destructively. Areas with malachite are artificially colored with red for the purpose of facilitating fication of malachite pigment

masterpieces, 'The Baptism of Christ'. the early 1600 s, the painting in a complex scene involving both the heavens and the earth, where Christ stands with St John the Baptist at his right. Strikingly, a number of figures are robed in bright greens, reds and yellows, giving a small indication of the complexity of the chemistry occuring on the campus.

The goal of the project coordinated by the Benaki Museum was to provide technical information to art historians to allow them to date and authenticate the painting and to determine what the physical condition of the painting During this process, the team foun a number of surprising results that only hyperspectral imaging could have unearthed, including the numerals 'MDLXVI', indicating the year 1566. This date had gone previously unnoticed as it had been overpainted, but the quality of the spatial resolution from the imaging made it possible to unambiguously resolve most of the date.

There was one small complication to this, though. Next to MDLXVl appeared to be anohther I, which would indicate the year to be 1567. Most other analysis techniques would have suggested the additional i"like feature was intended to be part of the date, owing However, the chemical

Professor Balas's interest in hyperspectral imaging was originally motivated by his work in the field of cancer diagnosis.

extracted from the hyperspectral imaging indicated this 'I' was not a numeral as part of the date, but actually intended to be part of a nearby scene.

colours and the techniques used to paint them often provide art historians with . depth chemical analysis of exactly which compounds were used where.

While hyperspectral imaging may be more efficient than point-by-point spectroscopic methods, the size of the painting posed some problems for Professor Balas and his team. They were able to identify over ten differe pigments used by El Greco and his lapis lazuli and azurite, but needed a more efficient way of identifying them over the full scale of the canvas. To do this, they turned to machine learning methods that, once they had been trained to identify the spectroscopic signatures of the various pigments, could then automatically assign which pigments had been used to paint specific regions, speeding up the process immensely.

\section{CAPTURING THE PAST} While not all paintings have dates conveniently hidden under layers of paint, hyperspectral imaging can be used to date paintings through a different approach. When chemists and artists this was often in to create new pigments this was ofen to to cate new
Hyperspectral imaging can be used not just for identification and dating but also for historical preservation. In cases where restoration is not possible due to the fragle nature of the object, . One example is the Codex Sinaiticus, the erliest manucript containing the complete New Testament which dates from the mid-fourth-century.

The Codex Sinaiticus is currently kept in four different locations, so as well as historical preservation, the goal of imaging the whole text also served the purpose of allowing a reunification of the complete text. The reunification of the text through imaging gave some ideas as to how the book may have now have a wealth of information on inks used in the text, all of which have in hyperspectral imaging. originally been bound and researchers in compositin of the dirferent coloured

\section{Behind the Research}

\section{Professor Costas Balas}

E: balas@electronics.tuc.gr T: + 3028210 37212 W: www.linkedin.com/in/costas-balas-b4473236/

\section{Research Objectives}

Professor Balas uses and develops hyperspectral imaging Prechnology in

\section{Detail}

\section{Costas Balas PhD}

Professor I Pirector Electronics Lab I School of Electrical \& Computer Engineering

University Campus Acroterion 73100,

Chania, Crete

Greece

Bio

Costas Balas is a full Professor at the Electrical and Computer Engineering department of the Technical University of Crete. He is a recognised expert and innovator in biophotonics and hyperspectral imaging. He has patented and published life-saving, FDA-approved photonic methods and imaging technologies for non-destructive analysis and for noninvasive diagnosis. He teaches both graduate and undergraduate courses.

\section{Collaborators}

The guidance and the data interpretation offered by Hadinich graduct students are grateflly acknowledged.

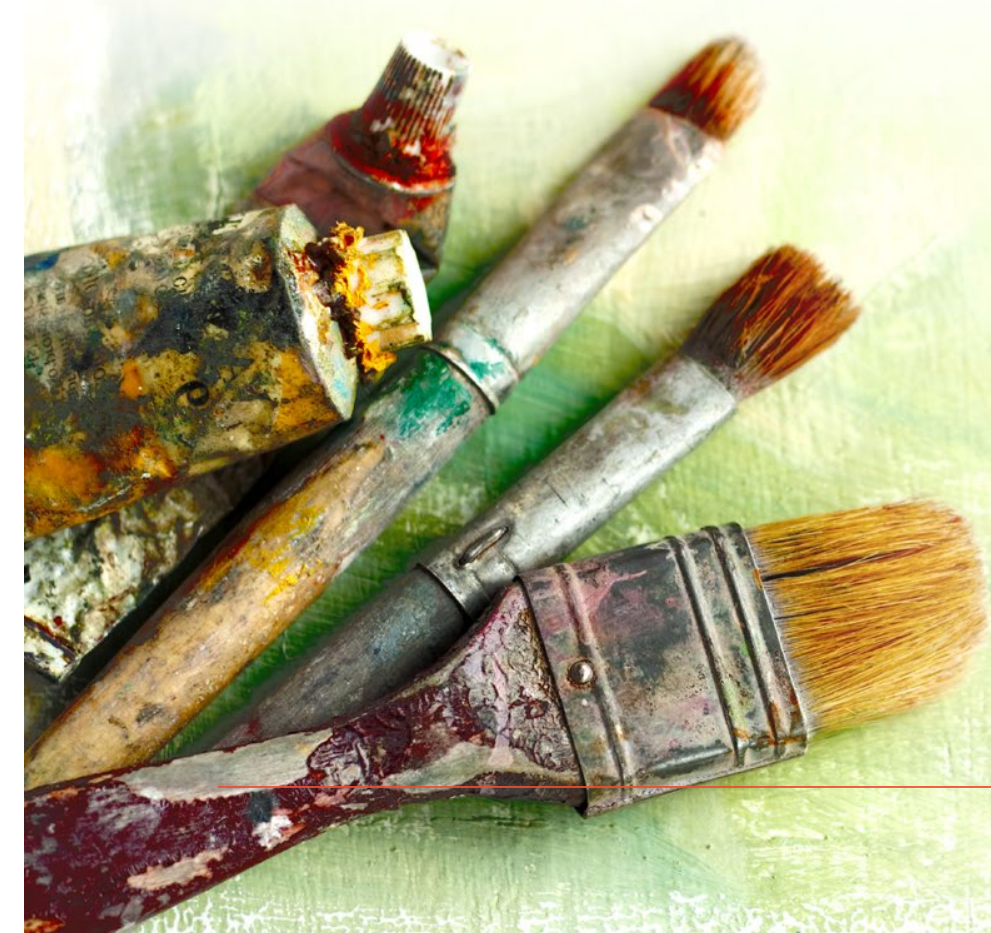

\section{References}

Balas, C., Epitropou G., Tsapras A. and Hadjinicolaou N., (2018) Hyperspectral imaging and spectral classification for pigment identification and mapping in paintings by El Greco and his workshop, Multimedia Tools and Applications, 77

\section{Personal Response}

What are the unique challenges of imaging art pieces? II Art pieces are very complex in nature with multiple layers and chemicals contributing to image formation. materials are often unktic art pieces, construction difficult to develop material replicas to be used as (n)

Innovative platforms integrating hyperspectral imaging
and machine learning methods emerge as a powerful tool for addressing these challenging diagnostic/ analytical tasks.

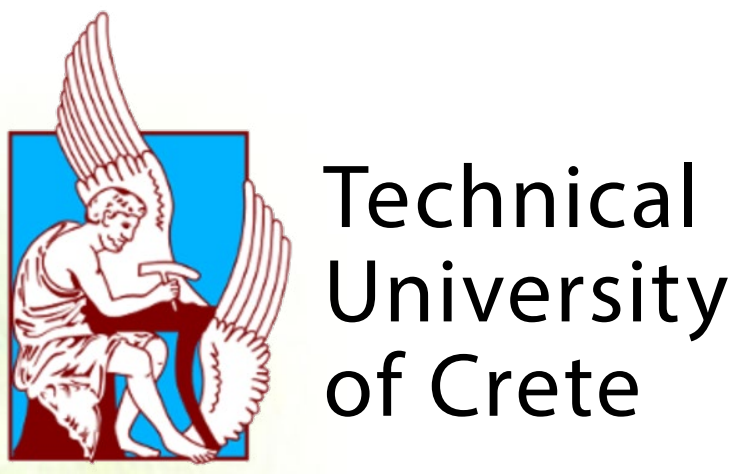

